\title{
La variabilidad histórica de la corriente del Río Colorado. El vínculo con la minuta 319
}

\section{The historical variability of the Colorado River current. The link with draft 319}

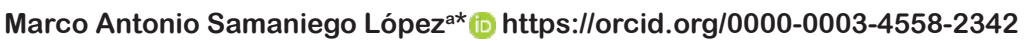

Recibido el 23 de mayo de 2016. Aceptado el 17 de mayo de 2017.

*Autor para correspondencia: Marco Antonio Samaniego López, correo electrónico: samaniego@ uabc.edu.mx

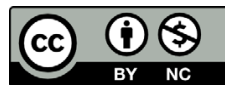

Esta obra está protegida bajo una Licencia Creative Commons Atribución-NoComercial 4.0 Internacional.
${ }^{a}$ Universidad Autónoma de Baja California, Instituto de Investigaciones Históricas, México, correo electrónico: samaniego@uabc.edu.mx

\section{Resumen}

El propósito de este artículo es analizar los efectos de la variabilidad histórica de la corriente del río Colorado y cómo se ha utilizado dicha variabilidad en las discusiones para el control y administración de la mencionada cuenca internacional. Con base en el análisis de estudios realizados por diversos ingenieros, abogados y políticos estadounidenses, demostramos que desde principios del siglo xx se sabía de esta variabilidad. Específicamente se busca explicar el significado y consecuencias que tuvo el tema de la variabilidad en la minuta 319, firmada por la Comisión Internacional de Límites y Aguas (International Border and Water Commission), donde por primera ocasión se contempla de manera explícita el cambio climático, dado que diversos sectores han insistido en profundizar en el tema de definir "sequía extraordinaria", argumentando que una cuota fija a México no tomó en cuenta el factor de cambio climático.

Palabras clave: cuenca transfronteriza, zona sísmica, cooperación-conflicto, variabilidad.

\section{Abstract}

The purpose of this article is to analyze the effects of the historical variability of the Colorado River current and how this variability has been used in the discussions for the control and administration of the mentioned international basin. Based on the analysis of studies carried out by various us engineers, lawyers and politicians, we have shown that since the beginning of the 20th century this variability was known. Specifically, it seeks to explain the meaning and consequences of the issue of variability in minutes 319 , signed by the International Boundary and Water Commission, where for the first time, since several sectors have insisted on deepening the issue of defining "extraordinary gear", arguing that a fixed quota for Mexico did not take into account the climate change factor.

Keywords: transboundary basin, seismic zone, cooperation-conflict, variability.

CÓMO CITAR: Samaniego, M. A. (2017). La variabilidad histórica de la corriente del Río Colorado. El vínculo con la minuta 319 [The historical variability of the Colorado River current. The link with draft 319]. Estudios Fronterizos, 18(37), 81-102, doi:10.21670/ ref.2017.37.a05 


\section{Introducción}

La minuta 319 firmada en noviembre de 2012 por la Comisión Internacional de Límites y Aguas, secciones mexicana y estadounidense, ha sido motivo de comentarios vinculados a efectos positivos en torno a posibilidades de futuro en la cuenca internacional del río Colorado (Minute No. 319 of the International Boundary and Water Comission, 2012). En documentos oficiales y algunos organismos ambientalistas que apoyan dicha minuta, se ha mencionado incluso que fue una renegociación del Tratado de Aguas Internacionales de 1944. Un tema que ha sido mencionado en la prensa son los beneficios del flujo base y flujo pulso en el ecosistema mismo que ha mejorado algunas zonas de la sección mexicana del bajo delta (Haro, 2017). El sustento se encuentra en diversas aportaciones de investigación sobre el delta desde, cuando menos, la década de 1990 (Glenn, Felger, Burquez y Turner, 1992; Glenn, GarciaHernández, Congdon y Luecke, 1999; Glenn, Lee, Felger y Zengel, 1996; Glenn et al., 1998; Luecke et al., 1999; Valdés-Casillas et al., 1998).

Como contraparte se han generado también acciones y críticas en sentido adverso a la firma de dicha minuta. Organizaciones de agricultores del Valle de Mexicali afectados por no tener forma de realizar sus cultivos han cuestionado la minuta ("Forman Frente Común", 2013). En 2013, 778 agricultores señalaron a los medios de comunicación que desde 2010 no habían recibido agua. Incluso, la legislatura estatal de Baja California realizó un exhorto al Senado de la República para la realización de una mesa de trabajo (Dictamen de acuerdo del congreso, 2013). La respuesta fue en el sentido de que no era de su competencia la revisión de dicha minuta (Boletín 1398, 2013). Sin embargo, se dirigió un exhorto al entonces secretario de Agricultura, Ganadería, Desarrollo Rural, Pesca y Alimentación, Enrique Martínez y Martínez y al también entonces director general de la Comisión Nacional del Agua, David Korenfeld para que se abasteciera a los agricultores. En agosto de 2015, en representación de 250 agricultores de la Central Campesina Cardenista, Max Correa, indicó que se presentaría una demanda ante el Tribunal Latinoamericano del Agua contra la Comisión Internacional de Límites y Aguas (CILA) por "actos de corrupción en el manejo del líquido" (Muñoz, 2015).

Uno de los puntos más importantes de la minuta 319 es el referente a las medidas proactivas en torno a los posibles efectos del cambio climático. Se afirma, sobre todo por académicos, instituciones y organizaciones ambientalistas, que la estimación sobre el caudal de la corriente realizada en la década 1910-1920, registró mayores niveles de humedad que en décadas posteriores (Robison et al. 2014, p. 18). En la minuta 319 se asienta que ambos países reconocen el valor de un periodo interino en el cual, de manera proactiva se "maneje el Río Colorado a la luz de la variabilidad histórica y su potencial incremento futuro debido al cambio climático". Con base en ello, se realizaron propuestas de modificación de las entregas a México, tema que tiene una larga tradición.

En los últimos 60 años de manera reiterada se indica que en el tratado no se definió en qué consiste la "sequía extraordinaria" (Hundley, 1963, p. 167). Dado que supuestamente no se realizó dicha definición, ante las prospectivas de un siglo xxI seco (Cook, Ault y Smerdon, 2015) el tema se vuelve importante en función de una necesaria cooperación binacional. Ante ello se han generado gran cantidad de textos con el fin de que ambos países propicien medidas alternativas ante una condición de la naturaleza que parece irremediable: el cambio climático y sus consecuencias en los 
procesos productivos, el abasto urbano, la producción de energía eléctrica, la pesca y su relación con la conservación del medio ambiente (Christensen y Lettenmaier, 2006).

En diferentes medios de comunicación se ha establecido un vínculo que conlleva a una relación que consideramos debe ser matizada: la variabilidad del flujo del río Colorado se debe al cambio climático. Numerosos autores e investigaciones del Bureau of Reclamation han publicado en los últimos lustros resultados de proyecciones a futuro sobre la disponibilidad de agua en las siguientes décadas (Barnett et al., 2004).

En este contexto, el propósito de este artículo es demostrar que la variabilidad del río Colorado ha sido argumento utilizado desde el inicio del siglo xx para justificar 1) el apoyo para la construcción de presas de propósito múltiple, 2) intentar obstaculizar el tratado de 1944 con México, 3) la promoción del trasvase de agua de otras corrientes como el Columbia, 4) el intento de modificar las condiciones del pacto de Santa $\mathrm{Fe}$ de 1922, ${ }^{1}$ o 5) como en el caso de la minuta 319, la negociación de reducciones de acuerdo con las posibles condiciones por el calentamiento global (Overpeck et al., 2013; Squillace, 2012).

El proceso de construcción de los tratados de 1906 y 1944 fue realizado con base en una idea de futuro enmarcada en el conservacionismo que triunfó sobre el preservacionismo en Estados Unidos (Samaniego, 2006, 2015). En ese contexto el objetivo era que el recurso hídrico no se fuera al mar: era un desperdicio que se debía evitar a toda costa y se debía aprovechar en la producción de energía eléctrica (Blanchard, 1924; Gray, 1913). Dicha concepción de la relación con la naturaleza impactó en la decisión de que toda el agua - futura y a perpetuidad- de los ríos Bravo y Colorado se asignara a los estados de las cuencas con la proyección de uso a grandes distancias y en estrecha relación con la producción de energía eléctrica. La "sequía extraordinaria" a la que se refieren en ambos tratados está ubicada durante el periodo de un año. La base de las negociaciones y proyecciones se realizó en acre-pie, medida inglesa que estima la cantidad de agua a la altura de un pie durante un año y que se consideraba la adecuada para la generalidad de los cultivos. Ese fue el eje de todas las negociaciones y es un concepto que se encuentra fuertemente vinculado con el de "sequía extraordinaria", es decir, en los tratados no se definió el concepto porque está vinculado directamente a una idea anual.

En el presente escrito destacamos que en la minuta 319, así como en la prensa que hace comentarios al respecto, se vincula la variabilidad de la corriente con el cambio climático; sin embargo, la corriente ha sido variable desde que empezó el registro de su caudal a finales del siglo xIx (Birdsong, 2011, p. 118; Bureau of Reclamation, 1946; La Rue, 1916). La variación del caudal fue uno de los argumentos para la construcción de presas. De igual forma, para tratar de detener el tratado con México o para justificar la supuesta ambigüedad sobre la calidad del agua del río Colorado o para la minuta aquí referida. Por ello, aunque de manera breve, ubicamos la firma de las minutas 317, 318 y 319, como parte de acuerdos en los que México realizó las negociaciones presionado por los efectos del temblor del 4 de abril de 2010, mismo que afectó los canales de irrigación en el Valle de Mexicali y se requería el apoyo estadounidense para conservar el recurso en el sistema hidráulico del vecino país. Consideramos oportuno mencionar que en lo referente a la minuta 319 existen otros puntos que son de suma importancia, sin embargo para los efectos de este escrito, solo tratamos el aspecto mencionado.

${ }^{1}$ El pacto de Santa Fe es la división que se realizó para la distribución del recurso del río Colorado. Dicho pacto, de manera no oficial, otorgó cierta cantidad de agua a México. 
En el presente escrito abordamos el tema de manera cronológica. Primero, las referencias sobre la variabilidad del río que datan de los primeros años del siglo xx. Posteriormente, demostramos que el argumento de la variabilidad se utilizó para justificar obras como la presa Hoover y el Canal Todo Americano. Luego, durante las arduas negociaciones en el senado de Estados Unidos, el mismo argumento se utilizó para tratar de detener la aceptación por los respectivos senados. En la década de 1960 sirvió para generar un proyecto que transfiriera agua del Río Columbia al Colorado y en los años recientes, ha sido vinculado al tema del cambio climático. Es decir, la variabilidad de la corriente se ha utilizado de diversas formas para provocar cambios en la manera de distribuir el recurso hídrico.

\section{Breve reflexión sobre el río Colorado}

El río Colorado es una corriente de carácter internacional que comparten México y Estados Unidos. En Estados Unidos corre de noreste a suroeste, por los estados de Wyoming, Utah, Colorado, Nevada, California, Nuevo México y Arizona, con una extensión de casi 2300 kilómetros. El área total de la cuenca es de 484330 kilómetros cuadrados, de los cuales 3840 se encuentran en territorio mexicano y el resto en los Estados Unidos. En términos porcentuales $99.28 \%$ corresponden al país vecino y apenas $0.72 \%$ a México. Como se puede observar, prácticamente la totalidad se localiza en el país vecino (Colorado River Development, 1928, p. 150-213).

En la actualidad y sobre todo por el atentado ocurrido el 11 de septiembre de 2001 en la ciudad de Nueva York, este cuerpo de agua es observado como un asunto de seguridad nacional tanto en México como en Estados Unidos (Cortez, Whiteford y Chávez, 2005) debido a que alrededor de 40 millones de personas dependen, bajo diversos usos sociales, del recurso hídrico. El Colorado es fuente de abasto de agua para usos urbanos de ciudades como Los Ángeles, California; Denver, Colorado; Phoenix, Arizona, así como valles agrícolas en México y Estados Unidos. Entre los más grandes podemos mencionar el Valle Imperial, en California y el Valle de Mexicali, en Baja California, México. De igual forma la corriente se utiliza para la generación de energía eléctrica, la pesca y el turismo. Solo en California, Estados Unidos, 17 millones de habitantes aprovechan de una forma u otra el recurso. Una ciudad como Tijuana, en México, con poco más de 1500000 de habitantes depende en $98 \%$ para su abasto de la corriente del Colorado que se localiza a 180 kilómetros de distancia.

Una de las condiciones naturales en la parte baja de la cuenca internacional del río Colorado es su aridez. La existencia de grandes zonas desérticas contrasta con la presencia de una corriente que durante muchos años fue una barrera natural para el movimiento de personas y mercancías, pero que con la implementación de avances en la tecnología hidráulica en el siglo xx se logró su control y administración. Se trataba de, en palabras del secretario del interior Hubert Work, convertir al gran río de una "amenaza natural" en un recurso nacional (Work, 1925). Esto generó grandes transformaciones en la manera de aprovechar la corriente, poblar grandes zonas y construir, por ejemplo en la sección baja del delta, —a pesar de las temperaturas en verano que llegan a los 50 grados centígrados - una región altamente productiva estrechamente relacionada con los mercados internacionales. 


\section{La variabilidad como constante: Argumento para diferentes fines}

Numerosos autores afirman que hubo optimismo en la firma del pacto de Santa Fe de 1922 y que se sobreestimó la cantidad de agua de la corriente (Adler, 2008, p. 30; Birdsong, 2011, p. 118). Sin embargo, el estudio de E. C. La Rue, que sirvió de base para analizar el potencial de la corriente o las declaraciones de representantes de entidades como Arizona, no apoyan tales afirmaciones. Algunos en Estados Unidos pretendieron generar la idea de que había agua suficiente para todos, como el director del Reclamation Service, Arthur P. Davis, o el entonces secretario de comercio Herbert Hoover. Sin embargo, el representante de Arizona W. S. Norviel, al escuchar los comentarios preguntó que si había agua suficiente para todos, porqué se establecían restricciones sobre la cantidad de flujo (Colorado River Commission, 1922).

El ingeniero E. C. La Rue señaló en su escrito que los resultados eran aproximados y que la demanda podría sobrepasar la cantidad existente: no había agua suficiente para todas las tierras que era posible cultivar. En el cuadro que publicó, el rango fue de 7960000 de acre-pie, en 1902, hasta un máximo de 25300000 acre-pie, en 1909. El promedio para los años 1895 a 1914, fue de 16200000 acre-pie (La Rue, 1916, p. 192). El autor del informe señaló que de construirse derivaciones alejadas del curso del río que no permitieran el retorno de agua, como fue el caso, se reduciría el caudal. De igual forma estimó las pérdidas por evaporación en las posibles presas que se construyeran en alrededor de 1000000 de acre-pie por año (La Rue, 1916, p. 167-169). Las conclusiones de La Rue fueron un referente constante para los planes subsecuentes (Colorado River Development, 1928, p. 150-213), así como uno de los más criticados por quienes afirman que se hizo mal el cálculo. En otro informe, publicado en 1925, afirmó que la corriente del río se modificaría cuando las obras que se planeaban realizar — 13 en ese momento- se efectuaran (La Rue, 1925, p. 9).

F. E. Weymouth, quien realizó el estudio para la construcción de la presa Boulder como jefe de ingenieros del Reclamation Service, fue otro de los más citados en las audiencias sobre las presas a construir. Ese año, en un informe presentado ante el senado de Estados Unidos, escribió en uno de los apartados:

Inundaciones rápidas arriba de Valle Imperial.

El río Colorado está sujeto a periodos de grandes inundaciones y grandes sequías. Se sabe que alcanza un máximo de descarga de más de 200000 pies cúbicos por segundo y una baja de corriente para el sistema de Imperial de 1250 pies cúbicos por segundo (F. E. Weymouth en Colorado River Develompent, 1928, p. 75)..$^{2}$

Así, el comité de estudios de irrigación y reclamación de tierras áridas del senado estadounidense, indicó que la cantidad de agua cambiaba año con año. Señaló que el promedio era de 17 millones de acre-pie. Afirmó de acuerdo con la visión del

${ }^{2}$ El original en inglés dice:

The Colorado River is subject to periods of great floods and great droughts. It has been know to reach a maximum discharge of more than 200,000 cubic feet of water per second and a low flow at the headworks of the Imperial system of 1,250 cubic feet of water per second (Colorado River Development, 1928, p. 75). 
conservacionismo que era necesario evitar el desperdicio por lo que era necesario eliminar que el agua se fuera al mar (Boulder Canyon Reclamation Project, 1926).

Entre las razones de quienes apoyaron la construcción de la mencionada presa era precisamente la variabilidad de la corriente. Un ejemplo es Clyde L. Seavey, quien publicó en 1928 un artículo titulado "What the Boulder Dam Project Means to California and to the Nation" en apoyo a su realización. Argumentó que como resultado de los cambios de caudal que se presentaban cada año, era necesaria la obra con la finalidad de regular la corriente, producir energía eléctrica y evitar que el agua se desperdiciara en el mar (Seavey, 1928).

Sobre el pacto de Santa Fe de 1922 se han escrito una gran cantidad de textos (Hundley, 1975; Olson, 1926). En dicho documento quedaron estimaciones tentativas de la corriente del río Colorado: 17000000 de acre-pie y con los excedentes 18000000 de acre-pie. Cabe señalar que La Rue nunca mencionó la segunda cantidad. En el mencionado pacto se asignaron siete millones y medio para las entidades superiores de la cuenca y la misma para los inferiores, California, Arizona y Nevada. En caso de excedentes, los tres últimos mencionados, podrían utilizar un millón más. De igual forma se contempló cómo se entregaría el agua a México en caso de que se realizara un tratado. Los estados superiores y los inferiores, (cuenca alta y cuenca baja, respectivamente) debían repartirse por igual la cantidad que se consideraba podría ser la proyectada: 1500000 acre-pie para México.

Las presiones de los estados de la cuenca eran diversas, contrapuestas y con posibilidades interrelacionadas. Es imposible en un escrito como este señalarlos todos. Sin embargo es importante destacar que la cifra se anualizó con el fin de establecer un parámetro para negociar lo que todos deseaban: la posibilidad de desarrollo futuro. Es decir, las cifras mencionadas estaban en el papel y a México llegaron hasta varios años después cantidades que superaban lo estipulado. Las obras a las que haremos mención en páginas posteriores redujeron sistemáticamente dicha cantidad. ¿Cuáles eran las presiones que existían en los años previos al pacto de Santa Fe? A continuación solo mencionamos algunos puntos que consideramos ubican el proceso de negociación.

1. La apertura de tierras en el bajo delta, es decir, los valles de Imperial y Mexicali, en California y Baja California, respectivamente, tuvieron éxito agrícola. Si se aplicaba el principio de primera apropiación era una amenaza para los estados superiores de la cuenca, ya que se podrían reclamar grandes volúmenes de agua.

2. La concesión otorgada por Porfirio Díaz en mayo de 1904 a través de empresas mexicanas formadas por estadounidenses, daba a nuestro país un total de 3600000 acre-pie. Valle Imperial, California, dependía de territorio mexicano y la única forma de eliminar parte de dicha dependencia era con la construcción del Canal Todo Americano.

3. Los representantes de la ciudad de Los Ángeles habían mostrado su interés por el recurso hídrico del río Colorado y dadas las prácticas en sitios como Owens Valley y Mono Lake ${ }^{3}$, iniciaron una campaña tanto para construir un acueducto desde el río Colorado como también para abastecerse del río San Joaquín, en el norte de estado. Los representantes de Colorado, Utah, Nuevo México y

${ }^{3}$ En Owens Valley y Mono Lake, se compraron derechos de agua para abastecer a Los Ángeles por un acueducto. Para el caso de Owens Valley significó una transformación de actividades agrícolas en ganaderas. El movimiento de resistencia de los agricultores tuvo momentos muy álgidos en la década de 1920. 
Arizona, consideraban como peligroso para su futuro las acciones de los representantes de dicha ciudad.

4. Todas las entidades tenían el temor de que se aplicara un embargo, como sucedió en el Alto Río Bravo y se restringiera el aprovechamiento a los usuarios de la cuenca. Dicha medida se implementó de 1896 a 1925 y prohibieron aprovechamientos en Colorado y Nuevo México hasta conocer los efectos de la presa Elephant Butte, obra construida luego del tratado de 1906. En 1911, California y Arizona lograron detener la construcción de obras en el estado de Colorado (National Archives Denver, s.f.). ${ }^{4}$

Bajo este marco, que es aún más complejo, sobre todo por la disputa entre empresas privadas que deseaban aprovechar la producción de energía eléctrica, se estableció la cifra de 15000000 de acre-pie, entre los estados de la cuenca, con el estimado mayor ya mencionado, 16200000 y 18000 000. Sin embargo, como hemos apuntado, es resultado de discusiones y claroscuros entre un conjunto de actores que pugnaban por obtener el recurso a futuro, no una información segura con base en datos confirmados. La Rue había sido claro, las extracciones que no permitieran el retorno, reducirían el caudal. Los lagos que se formaran con las presas, debido a la evaporación, también (La Rue, 1916).

En el reporte del comité de irrigación del senado en 1926 se señaló que era necesario un acueducto a Los Ángeles y otros condados aledaños. Indicaron que en la costa del pacífico se vivían periodos de sequía de entre 10 y 12 años. Los bonos para el pago de la obra, por 2 millones de dólares ya habían sido autorizados (Boulder Canyon Reclamation Project, 1926, p. 21). Situaciones semejantes se habían registrado en Denver, Colorado, en la primera década del siglo xx. Estas extracciones, que se volvieron comunes en los siguientes años, no permiten los procesos de retorno y eran precisamente a las que se refería La Rue.

En diciembre de 1928 se aprobó la construcción de la presa Boulder. El objetivo declarado era el control de la corriente, producir energía eléctrica y entregar agua para usos agrícolas. Lo que más interesaba era proteger al Valle Imperial, California, de las inundaciones y modificar en lo posible el enorme problema de la salinidad que generaba el paso de la corriente por el Gran Cañón. De igual forma, bajo el principio de "primera apropiación", California garantizaba un mayor uso de agua del río. Con la obra, México sin duda obtenía grandes beneficios al reducirse significativamente el tema de las inundaciones, los sedimentos y aumentar la apertura de tierras que le permitieran reclamar la cantidad de agua que pretendía para las 200000 hectáreas proyectadas. Por otra parte, con la presa y la construcción del Canal Todo Americano, se perdió una ventaja en las negociaciones sobre las corrientes internacionales, dado que Valle Imperial ya no dependería de territorio de México (Samaniego, 2006).

La presa Boulder significó un gran triunfo de la ingeniería que se buscó replicar. Así, mientras se desarrollaba este proyecto se realizaron investigaciones y se aprobó The Colorado-Big Thompson Project, en el estado de Colorado. Dicha obra requería de producir energía eléctrica para que el agua ascendiera y luego, por gravedad, llegara al lado este de las montañas. Con ello, agua del río Colorado se retiraba de los procesos de retorno como ha sucedido en numerosos proyectos de años subsecuentes.

${ }^{4}$ El expediente National Archives Denver, Record Group 115 contiene numerosos documentos en los que organizaciones de California solicitan que no se otorguen concesiones de agua en las entidades superiores de la cuenca, como Colorado, Wyoming, Utah y Nuevo México. 
Un par de sequías en 1932 y 1934, así como la referencia sobre los beneficios en México propiciaron que los representantes de California y Arizona se opusieran a la cantidad de agua que pretendía nuestro país. Durante esos años la postura mexicana se modificó a reclamar 1500000 acre-pie anuales. California pretendía que se aprobaran 750000 y Arizona, absolutamente nada. Por su parte entre estas entidades se desarrollaron dos casos judiciales, ganados por California. Por ello, durante el proceso de ratificación del tratado, Arizona prefirió apoyarlo en contra de los deseos de California. Las entidades superiores de la cuenca, entre ellas Colorado, apoyaron el tratado con México. Como veremos más adelante, durante el presente siglo la actitud de Colorado se modificó sustancialmente.

\section{La variabilidad no permite el tratado: California}

Si Arizona se había opuesto al pacto de Santa Fe, California lo hizo con respecto al tratado de 1944. Los estados de la cuenca superior, Wyoming, Nuevo México, Utah y Colorado, que en términos comparativos tenían menos avances en el uso de la corriente, apoyaron la firma del acuerdo. Sin embargo, California, se opuso con varios argumentos. Aquí solo destacamos los relacionados con la cantidad del cuerpo de agua. Robert W. Kenny, fiscal general de dicha entidad, indicó que el Colorado era el más "relampagueante" e "impredecible" de los ríos del mundo. Su flujo más alto era de 25000000 de acre-pie en un año, pero recordó a los presentes en la audiencia del senado que en 1934 apenas se registraron 4400000 acre-pie. Por tanto, no se podían cerrar las compuertas de sus instalaciones para cumplir con los proyectos de extranjeros (Water Treaty, 1945, p. 373).

Philip D. Swing, señaló en su comparecencia ante el senado que los ingenieros consultados afirmaron que en las últimas dos décadas se había presentado una baja en la corriente del río. Swing señaló que el tratado con México garantizaba derechos y que era una ley suprema en el país y anulaba, en caso de que se ratificara, las leyes federales, estatales y los contratos, por lo que significaba que alguna comunidad "va a sufrir". No podía decir quien, Arizona, California, Utah, Nevada o Wyoming:

...Pero alguien va a ser herido, porque cuando acordamos entregar más agua de la que existe de acuerdo a los registros, sólo hay una conclusión que se puede dibujar, y esa es que ahí hay una deficiencia y alguien va a sufrir (Water Treaty, 1945, p. 413). ${ }^{5}$

Así, mientras en las discusiones para la aprobación de Boulder los californianos, y en específico Swing como coautor de la propuesta, utilizaron el argumento de la variabilidad para indicar que con la presa Boulder se podría regular la corriente y administrar el recurso de mejor manera, para la aprobación del tratado esta condición efectivamente variable era el argumento para no aprobar el tratado.

La comparecencia del ingeniero Clay C. Elder, como integrante del sistema metropolitano de Los Ángeles, sostuvo el mismo argumento. Sus afirmaciones como

\footnotetext{
${ }^{5}$ Indicó Swing: “...but somebody is going to be hurt, because when we have agreed to deliver more water than is according to the records, there is only one conclusion you can draw, and that is that there has got to be a deficiency and somebody has got to suffer" (Water Treaty, 1945, p. 413).
} 
experto en el área fueron en el sentido de que el Bureau of Reclamation, no tenía forma de saber cuánta agua sería utilizada en función del tipo de proyectos que se aprobaran, sobre todo por los estados de la cuenca alta —Colorado, Utah, Wyoming, Nuevo México-. Además de mencionar las diferencias de caudal, semejante a los datos proporcionados por La Rue, afirmó que una prueba de que la corriente variaba significativamente fue la década de 1930 y particularmente el año de 1934. Los contratos firmados para producción de energía eléctrica no se podrían cumplir y los datos proporcionados por ingenieros de diversas empresas confirmaban el hecho de que cada gota de agua estaba asignada. Mencionó que estados como Arizona y California otorgaron contratos de entrega de agua y energía durante esos años a pesar de que las obras estaban muy lejos de realizarse (Water Treaty, 1945, pp. 463-480).

Ante preguntas del comité del senado sobre los sedimentos, la calidad del agua y los retornos, Elder dejó en claro que los datos no eran precisos por más que se intentara demostrar cómo se enfrentarían dichos retos. Elder indicó que cuando se presentaran las sequías extraordinarias como en el pasado, los usos ya repartidos a pesar de no existir las obras, cambiarían el sentido de las aguas de retorno. En su larga presentación, mantuvo el argumento del error del senado de aprobar el tratado y señaló, desde entonces, uno de los argumentos que se han repetido en las últimas décadas del siglo xx e inicios del xxi: con el método de los tres anillos era posible señalar que en siglos precedentes se habían registrado grandes sequías, como en el $\mathrm{XVI}^{6}$ (Water Treaty, 1945, p. 490).

De esta forma, si californianos habían argumentado que la presa Boulder solucionaría el problema del control de la corriente por su variabilidad, ante la firma del tratado con México se sustentaban en argumentos semejantes a los destacados por los representantes de Arizona. Phil Swing, que años antes argumentó que la presa Boulder era indispensable por la variabilidad de la corriente, ante el tratado con México, utilizó dicho argumento para intentar que este no se aprobara. Sin embargo, los representantes californianos fracasaron en su intento y el tratado fue aceptado en el senado de los dos países (Enríquez, 1976; Hundley, 1975; Samaniego, 2006).

\section{Ante un río variable, se necesita agua de otro río: El Columbia}

Posterior a la aprobación del tratado con México ratificado por los senados de los dos países en 1946, se iniciaron los proyectos para el desarrollo de las entidades localizadas en la cuenca alta. Colorado, Utah, Nuevo México y Wyoming, llegaron a un pacto en 1948 en el cual se repartieron el recurso que les correspondía de acuerdo a porcentajes. Desde 1950, algunos autores indicaban que el problema era que los proyectos habían sobrepasado las posibilidades reales de la corriente (Parsons, 1950, p. 47). De igual forma, los actores del conservacionismo empezaron a actuar diferente con respecto al medio natural. Para el caso del río Colorado, fue el inicio de propuestas en contra de las presas que por un lado se llenaban de sedimentos y por otro el cuidado del Gran Cañón.

\footnotetext{
${ }^{6}$ El método es conocido también como dendocronología. De manera sintética señalamos que a partir de los anillos en los troncos de los árboles se realizan interpretaciones sobre las sequías o los periodos de abundancia de agua, entre otras cosas. El desarrollo de esta área de la ciencia surge en el siglo XIX, por lo que se aplica desde hace más de un siglo.
} 
Por su parte, Arizona mantuvo los conflictos y demandas en contra de California y estos, a su vez iniciaron procesos de oposición al desarrollo de los estados superiores de la cuenca. Uno de los argumentos fue precisamente la variabilidad del río y la amenaza de no poder cumplir con los contratos y entregas en un futuro con crecimiento poblacional constante, en una proyección para el año 2030. Por ello surgió un tema que ha sido retomado en varias ocasiones en los años posteriores: de los 160 millones de acre-pie del río Columbia, ${ }^{7}$ con un porcentaje mínimo sería posible resolver el problema del agua en las entidades de la cuenca del Colorado. Este objetivo es la razón de las proyecciones que se realizaron en las audiencias para la aprobación del Central Arizona Project (CAP) mismo que fue motivo de intensas negociaciones desde 1949 hasta su aprobación en 1968. Las audiencias de 1965 que aquí citamos tuvieron un propósito: solucionar el tema de los inevitables recortes que se proyectaban para fines del siglo xx y la única forma de hacerlo era con agua del Columbia.

¿Por qué la alarma a mediados de la década de 1960 en su proyección hacia 2030? Anotamos los siguientes puntos, pero señalamos que son una síntesis de una problemática de mayor complejidad y detalle.

1. La decisión del caso Arizona vs California (1963, 373 U.S. 546) reconoció el derecho de Arizona de utilizar los 2800000 acre-pie señalados en The Boulder Canyon Act de 1928. El Gila quedaba para uso exclusivo de dicha entidad. California consideraba que perdía 700000 acre-pie, obtenidos en 1929.

2. Vinculado estrechamente con Arizona vs California (1963, 207 U.S. 564) resurgió el tema de los derechos de los pueblos indígenas. En el laudo se retomó la decisión Winters de 1908, que no había impactado hasta ese momento el reparto del agua del río, pero desde 1963 es un factor de peso en las discusiones, demandas en las cortes, proyecciones y venta de derechos de agua.

3. El incremento de actividad y decisiones judiciales en torno a nuevas formas de conservacionismo y medioambientalismo. Destaca la presa Glen Canyon, misma que se convirtió en el símbolo de los daños al ambiente (Farmer, 1996, p. 210; Harvey, 1991). Posterior a ello, se aprobaron, entre otras The Clean Water Act, la National Enviromental Policy Act y la Endangered Species Act.

4. California utilizó más agua que lo aprobado en The Boulder Canyon Act y la Limitation Act de 1929, con un promedio de 5100000 acre-pie. ${ }^{8}$ Desde la década de 1960 se ideó el plan 4.4 para limitar el uso de California a lo indicado por la Boulder Act de 1928. Sin embargo, el plan 4.4 se inició hasta 1999 y es una de las presiones actuales, sobre todo porque se contempló a quince años, es decir, para el 2015.

5. Las entidades superiores de la cuenca y especial el estado de Colorado, insistieron en el uso excesivo de California. En este contexto surgió la propuesta que han sostenido desde hace algunos años, en el sentido de que no es su responsabilidad la parte que le corresponde a México de acuerdo al tratado de 1944 y en relación con lo previsto en el pacto de Santa Fe de 1922, en el cual se afirma que la cantidad asignada a México en caso de no haber más de 15 millones de

\footnotetext{
${ }^{7}$ Dicho río es de carácter internacional. Surge en Canadá y desciende a Estados Unidos. Los estados de la cuenca son Washington, Oregon y Idaho. Algunos afluentes corresponden a Montana, Wyoming, Utah y Nevada. Es el cuarto más caudaloso de Estados Unidos y desemboca en el Océano Pacífico.

${ }^{8}$ En ocasiones los usos de California sobrepasaron los 7000000 de acre-pie.
} 
acre-pie, se cargará por mitad a las entidades superiores y a las inferiores de la cuenca (Hearings before the subcomitte on irrigation and reclamation, 1965, pp. 288-290).

Bajo este escenario se ha mantenido hasta nuestros días la discusión sobre la cantidad de agua en el río Colorado. Es decir, la referencia acerca de la falta del recurso hídrico no apareció con el tema del cambio climático de las últimas décadas. Como ya apuntamos, en las audiencias del subcomité de irrigación del senado, de agosto-septiembre de 1965, se discutieron las proyecciones hasta 2030. El secretario del interior, Stewart L. Udall, de manera clara indicó que el agua del río Columbia era la solución permanente del problema (Hearings before the subcomitte on irrigation and reclamation, 1965, pp. 128-137). Udall, nativo de Arizona, era un declarado conservacionista (Smith, 1995). De igual forma, impulsó la realización del Central Arizona Project (CAP), mismo que estaba en discusión para su aprobación en la audiencia de 1965 Hearings before the subcomitte on irrigation and reclamation (1965). ${ }^{9} \mathrm{En}$ el contexto de las audiencias prefería la aprobación del proyecto que beneficiara su estado natal ante la oposición de California y Colorado, cuyos representantes reafirmaban la imposibilidad de cumplir con las demandas ya existentes y predecían recortes para la década de 1990 o el año 2000. Por ello el tema recurrente fue la importación de agua del río Columbia.

Stewart L. Udall argumentó la tendencia en el crecimiento poblacional de California, Colorado, Arizona y Nevada. Por ello era necesario reducir los usos de California. En ese contexto, Udall tomó el año de 1934 como ejemplo de las variaciones del caudal del río, cuando solo hubo 4.4 millones de acre-pie. Northcutt Ely, como representante de California indicó que la única posibilidad para solucionar los problemas de los cambios de caudal era con la importación de agua a la corriente principal del río. Udall apoyó lo sustentado con el fin de buscar la aprobación del cAP, de que en efecto, la solución era importar del río Columbia un porcentaje mínimo de los 160 millones de acre-pie que se iban al mar. Si Ely mencionó 2.5 millones de acre-pie como indispensables para cumplir con las obligaciones, Udall indicó que si se lograba la importación de 10 millones de acre-pie, aun así se desperdiciarían en el mar 150 millones del Columbia (Hearings before the subcomitte on irrigation and reclamation, 1965, pp. 312-318).

Para los efectos del presente escrito destacamos que el método de los tres anillos (dendrocronología) de manera semejante a dos décadas atrás —o en la actualidad—fue el sustento de la argumentación. Así, por ejemplo, Floyd E. Dominy, comisionado del Bureau of Reclamation, indicó que los estudios daban como promedio un total de 15 millones de acre-pie, aunado al problema de los periodos en que el caudal disminuía (Hearings before the subcomitte on irrigation and reclamation, 1965, p. 224). Sin embargo, otros datos fueron mencionados de acuerdo con los periodos que se seleccionaban y la cifra que también se utilizó fue que en realidad solo eran 13.2 millones de acre-pie como promedio.

En 1965 no se aprobó el CAP y fue motivo de negociaciones en los tres años siguientes. En audiencias de 1967, se presentó un informe por parte del Bureau of

\footnotetext{
${ }^{9}$ En los mismos años se inició el tema que se ha conocido como "la salinidad del río Colorado" que generó numerosas diferencias con nuestro país. Al respecto, en este escrito solo apuntamos que el tema requiere de un análisis más detallado, dado que se necesita hacer la distinción de que se trataba de agua que no correspondía al río, sino de aguas del subsuelo de Wellton-Mohawk.
} 
Reclamation, en un contexto semejante al ya descrito: lograr el traslado de agua del río Columbia. Además de apuntar que la variación era entre 5.6 millones de acre-pie y 25 millones, en el informe se indicó que sobre las variaciones naturales se había incrementado el agotamiento debido a los usos de la cuenca alta. Se afirmó que la proyección establecida en el pacto de Santa Fe de 1922 no se había registrado en las últimas décadas. Por tanto, los análisis que se presentaban en el informe estaban con base en los embalses construidos para maximizar el uso de la corriente (Hearings before the subcomitte on irrigation and reclamation, 1967, p. 94).

De esta forma, la variabilidad de la corriente se utilizó dentro de contextos de discusión que permitieran realizar obras para aprovechar de manera óptima el recurso hídrico en el marco de los enfrentamientos y colaboración entre cuencas y estados del vecino país. Así mismo, para amenazar, cuestionar y ponderar el tratado de 1944 con México.

\section{Un escenario sobre la variabilidad en el año 2009}

Las proyecciones de la década de 1960 plantearon retos que se han incrementado con las posibilidades analizadas sobre el cambio climático. En la primera década del siglo XXI se llegó a los niveles más bajos de reservas en las presas. Los modelos a largo plazo, algunos para el 2050, otros para el 2100, indican que se dará un incremento en la temperatura de la cuenca de $1.8^{\circ}$ a $4.5^{\circ}$ centígrados (Christensen y Lettenmaier, 2006). Por otra parte, las proyecciones de crecimiento de la población para todas las entidades de la cuenca indican que en los próximos años las tensiones para el abasto urbano se incrementarán notablemente (Nowak, Hoerling, Rajagopalan y Zagona, 2012).

A partir de las pruebas que han evidenciado desde la década de 1970 el impacto que tiene el desarrollo industrial y los cambios en el medio ambiente, las propuestas para tomar medidas y detener en lo posible el cambio climático se han incrementado. En 1988 surgió el Intergovernmental Panel of Climate Change (IPCC) como resultado de las acciones de diversos países sustentados en estudios científicos que se iniciaron en la década de $1950 .{ }^{10}$ Con ello el tema del cambio climático ha permeado a la sociedad global desde hace seis décadas. Para el caso del oeste estadounidense existen numerosos documentos que en las últimas décadas plantean los posibles efectos en una región reconocida como árida y sobredependiente del río Colorado (Fahlund, Choy y Szeptycki, 2014). Esto, por supuesto, tiene implicaciones directas en México.

$\mathrm{Si}$ en el apartado anterior apuntamos varias tensiones que se vivieron, en los años recientes se han mantenido varias de ellas y todas vinculadas con los apartados anteriores.

1. Los efectos del cambio climático en el suroeste estadounidense y en lo específico en las regiones interrelacionadas al río Colorado, se han vuelto una prioridad.

2. Desde las discusiones ya planteadas en las décadas de 1940 y 1960 la pregunta en la cual se centra la atención es cómo se resolverá el problema

\footnotetext{
${ }^{10}$ A partir de los estudios del científico estadounidense Charles Kelling (1928-2005) se generaron las primeras discusiones sobre el cambio climático, aunque dicho término surgió hasta 1979. Kelling quien hizo las primeras mediciones de dióxido de carbono (CO2) en 1958 en Mauna Loa, Observatorio Astronómico ubicado en la cima de un volcán inactivo de Hawai.
} 
cuando se presenten las sequías. De ahí se desprende con insistencia la inserción del tema de cómo definir la "sequia extraordinaria".

3. California inició la aplicación del Plan 4.4 hasta 1999, por lo que entidades como Colorado han reafirmado su postura de no entregar la parte correspondiente a México.

4. A partir del caso Arizona vs California, donde se retoma la decisión Winters de 1908, en las últimas décadas los grupos indígenas han incrementado sus pretensiones de agua. En varios casos han tenido éxito como fue el revestimiento del Canal Todo Americano (Sánchez, 2005).

5. Los avances de los grupos conservacionistas son notables. Provocaron que el Bureau of Reclamation reorganizara sus políticas y modificara los lineamientos para la producción de energía eléctrica, logrando que se sostengan flujos de agua para ciertas áreas.

6. Si bien el proyecto de trasladar agua del río Columbia no se ha realizado, tampoco se ha descartado (Hicks, 2007). En diversas notas periodísticas que van desde 1990 hasta 2015, el asunto reaparece (Simon, 1990; Kelly, 2015; Fox, 2015). A dicha propuesta se ha sumado la posibilidad de que agua del alto Missouri sea la que provea agua al Colorado.

Bajo este escenario, el tema del cambio climático y las posibles consecuencias tuvo un evento contingente que ha generado una modificación, cuando menos temporal, de la entrega de agua a México, misma que aludimos al inicio de este escrito: la minuta 319 .

\section{El terremoto de abril de 2010 y las minutas 317, 318 y 319. ¿Nueva forma de distribución del recurso? ¿Renegociación no reconocida del tratado de 1944?}

Una de las referencias constantes que involucra numerosos factores es que los estados de California, en Estados Unidos y la península de Baja California, en México, se localizan en la llamada falla de San Andrés (Cruz-Castillo, 2002; Shor, Russell y Mc Gowan, 1976). Se trata de una zona sísmica que de manera general tiene entre uno de sus lugares más identificados la ciudad de San Francisco, California y abarca hacía el sur toda la península de la Baja California, en México.

El cuatro de abril de 2010 se registró un temblor de 7.2 grados en la escala de Richter, mismo que afectó a California, Estados Unidos, así como a Baja California y Baja California Sur, en México. En los valles de Mexicali e Imperial, donde los temblores de tierra se presentan con mayor intensidad hubo varios efectos. Uno de ellos fue que los canales de irrigación que abastecen la zona agrícola de Mexicali resultaron dañados. Con ello, las entregas mensuales que se realizan en coordinación entre la Comisión Internacional de Límites y Aguas (CILA) y la International Boundary and Water Comission (IBWC, por sus siglas en inglés), de sostenerse de acuerdo a los calendarios establecidos, provocarían pérdidas del vital líquido y posibles inundaciones en cierta áreas.

Cabe señalar que desde el siglo xix la Comisión Internacional de Límites, —desde 1944 Comisión Internacional de Límites y Aguas— ha signado minutas para llegar a 
acuerdos sobre diversos aspectos. Para el tema que aquí nos ocupa, destacamos que las condiciones de los canales de irrigación luego del temblor colocaron a México en una situación de requerir la negociación. Los temas que estaban en la mesa eran varios, sobre todo los sostenidos por los conservacionistas que deseaban asignación de agua para restaurar zonas del bajo delta (Conway, Nadeau y Piest, 2010). En al año 2000 se firmó el acta 306 que respondía a propuestas de esa índole, pero en una década no se había avanzado en ello.

\section{La minuta 317}

En la minuta 317, signada el 17 de junio de 2010, se ratificó la importancia del trabajo conjunto. En ese marco, se dio un paso importante al reconocer las actividades de las organizaciones no gubernamentales. Con base en ello, se dio origen al denominado Grupo Consultivo Binacional. En dicho organismo, participan representantes de la propia comisión, los gobiernos federales así como de los gobiernos estatales de ambos países.

En la minuta se registró que ambas naciones deseaban explorar oportunidades para minimizar los posibles impactos de potenciales condiciones de escasez del río Colorado. No se mencionó el tema del cambio climático de manera expresa. Aunado a ello, se abordaron otros temas como búsqueda de nuevas fuentes de abastecimiento, mejoramiento de la infraestructura hidráulica, así como asignar recurso hídrico al mejoramiento del medio ambiente. Entre otros aspectos, se apuntó que se consideraría "la posibilidad de permitir a México el uso de la infraestructura estadounidense para almacenar agua" (Minute 317, 2010, p. 4).

\section{La minuta 318}

El 17 de diciembre de 2010 se signó la minuta 318, en la cual se ajustó el calendario de entregas a México, para los años 2010 a 2013. El fundamento fue el artículo 15, inciso f, del tratado de 1944, donde se menciona la posibilidad de cambiar la tabla mensual de entregas.

En el documento se asentó que el gobierno mexicano solicitaba la modificación debido a que 640 kilómetros de canales de irrigación resultaron afectados por el terremoto. Alrededor de 60000 hectáreas se anegaron o era imposible hacer llegar el agua a las parcelas. Por ello, de la cantidad perteneciente a México, —1 $850234000 \mathrm{~m}^{3}$ — se acordó que se retuvieran durante el periodo señalado 320 millones de $\mathrm{m}^{3}$, es decir, poco menos de una sexta parte del total. Esta cantidad se retendría hasta el 31 de diciembre de 2013, periodo considerado suficiente para reparar las obras de infraestructura en el Valle de Mexicali. En la minuta se reconoció la necesidad de minimizar los impactos en condiciones de escasez "ante el creciente reconocimiento sobre los potenciales efectos del cambio climático" (Minute 318, 2010, p. 4). Ambos países estuvieron de acuerdo en continuar con los esfuerzos para atenuar los impactos referidos. La diferencia entre la minuta anterior es que en esta se mencionó de manera clara el tema del cambio climático. 


\section{La minuta 319}

La minuta 318 fue firmada bajo un supuesto: los canales de irrigación en el Valle de Mexicali estarían listos para diciembre de 2013. Sin embargo, para mediados de 2012 era claro que no sería posible. Por ello, se signó el 20 de noviembre de 2012 la minuta 319, a diez días del cambio de administración federal en México. Conviene destacar que por primera vez se estableció un método distinto en lo referente a una parte de la cantidad de agua que México va a recibir hasta el 31 de diciembre 2017. Es decir, es una minuta que contempla cinco años, pero de funcionar para ambos países puede prolongarse hasta el 2026.

Esta consideración, ha sido motivo de comentarios y críticas dada su importancia en el futuro en la cuenca internacional del río Colorado. En este sentido, como ya apuntamos, en documentos formales y asociaciones ambientalistas que apoyan dicha minuta se ha mencionado lo amplio de su alcance y que incluso puede tratarse de una renegociación de facto del Tratado de Aguas Internacionales de 1944. Evidentemente la sola mención de este punto generó varias tensiones entre los agricultores del Valle de Mexicali (Arellano, 2013).

En la minuta 319 se tocaron otros temas de significación, que no abordaremos en el presente escrito. Nos limitamos al aspecto de las entregas de agua a nuestro país. Por ello destacamos que en la minuta se modifica el escenario de la cantidad que corresponde al tratado de 1944, dado que se toma como referencia la altura del lago Mead, formado por la ya mencionada presa Hoover. Ambos países, reconocieron el valor de un periodo interino de cooperación para que de manera proactiva se maneje el cuerpo de agua a "la luz de la variabilidad histórica y su potencial incremento futuro debido al cambio climático, por lo que dicho manejo proactivo maximizará la utilización del recurso durante condiciones de variabilidad de los almacenamientos en beneficio de ambos países" (Minute No. 319 Interim International Cooperative Measures in the Colorado River Basin, 2012, p. 3). De esta forma, como hemos apuntado en este escrito, la variabilidad del flujo de agua del río no es la primera vez que se argumenta para la toma de decisiones. Fue el sustento para construir las presas, para discutir la forma de distribuir el recurso, para oponerse al tratado con México o para intentar el trasvase de aguas del río Columbia. En esta ocasión se relacionó con el tema que aparece en la agenda mundial: el cambio climático.

De esta forma, en la minuta 319 quedó establecido que en el caso de que se registre un decremento, los efectos negativos sean proporcionales a todos los estados de la parte baja de la cuenca, es decir, California, Arizona y Nevada en Estados Unidos y en México, Baja California. Las entidades consideradas como ribereños superiores, Wyoming, Colorado, Utah y Nuevo México en Estados Unidos, no están involucradas en este acuerdo, dado que las modificaciones corresponden a aguas abajo de la presa Hoover. Dicho de otra manera, a partir de la innegable variabilidad de la corriente, de la que se tenía conocimiento desde principios del siglo xx y se dieron pronósticos de recortes de agua en las décadas de 1940 y 1960, fue posible modificar las entregas en relación directa a los efectos del terremoto de 2010. Como hemos apuntado, en principio es por cinco años, aunque se considera la posibilidad de su ampliación hasta 2026. Se menciona que se trabajará en una minuta integral, lo que es de suponerse ha sido motivo de discusión al interior de la CILA-IBWC. 
De acuerdo con lo establecido, las modificaciones serían de la siguiente forma; México puede recibir excedentes de acuerdo con los incrementos en la Presa Hoover (ver la Tabla 1).

Tabla 1: Incremento anual para México de acuerdo con la altura del lago Mead

\begin{tabular}{|l|c|}
\hline \multicolumn{1}{|c|}{ Elevación en el lago Mead } & Incremento anual para México \\
\hline $\begin{array}{l}\text { En o por encima de } 1145 \text { pies sobre el nivel del mar } \\
\text { (psnm) y por debajo de } 1170 \mathrm{psnm}\end{array}$ & $49 \mathrm{Mm}^{3}$ (40 000 acre-pies) \\
\hline En o encima de 1170 psnm y por debajo de $1200 \mathrm{psnm}$ & $68 \mathrm{Mm}^{3}$ (55 000 acre pies) \\
\hline $\begin{array}{l}\text { En o por encima de } 1200 \text { psnm y no se requieren descar- } \\
\text { gas de control de avenidas }\end{array}$ & $99 \mathrm{Mm}^{3}(80000$ acre pies $)$ \\
\hline $\begin{array}{l}\text { Cuando se requieren descargas de control de avenidas sin } \\
\text { importar la elevación }\end{array}$ & $247 \mathrm{Mm}^{3}(200000$ acre pies $)$ \\
\hline
\end{tabular}

Fuente: Minute No. 319 Interim International Cooperative Measures in the Colorado River Basin (2012).

En sentido contrario, se estipulan reducciones en el caso de falta de disponibilidad. Este tema es el que causa preocupación, sobre todo entre campesinos del Valle de Mexicali. En la minuta se mencionan las cantidades que se dejan de entregar para las entidades estadounidenses de la cuenca baja y de manera semejante, se establecen las reducciones para México; se toma como base el 1 de enero de cada año y se reduce como se observa en la Tabla 2.

Tabla 2: Reducción anual para México de acuerdo con la altura del lago Mead

\begin{tabular}{|l|c|}
\hline \multicolumn{1}{|c|}{ Elevación del lago Mead } & Reducciones a México \\
\hline Por debajo de 1075 psnm y en o por encima de $1050 \mathrm{psnm}$ & $62 \mathrm{Mm}^{3}$ (50 000 acre pies) \\
\hline Por debajo de 1 $050 \mathrm{psnm}$ y en o por encima de 1025 & $86 \mathrm{Mm}^{3}$ (70 000 acre pies) \\
\hline Por debajo de $1025 \mathrm{psnm}$ & $154 \mathrm{Mm}^{3}$ (125000 acre pies) \\
\hline
\end{tabular}

Fuente: Minute No. 319 Interim International Cooperative Measures in the Colorado River Basin (2012).

De esta forma, sin la aprobación formal de los senados de ambos países, se modificó la tabla de entregas que se definen en relación directa al almacenamiento de la presa Hoover. Si bien en reiteradas ocasiones se manifiesta en la minuta 319 su carácter temporal, no resulta suficiente para sectores campesinos que ven en ello un atropello de Estados Unidos a México. Como ya apuntamos, el tratado de 1944 prevé reducciones por consumos, no por caudal. Es uno de los referentes que se han constituido en un motivo de controversia dada la interpretación que se le da al tema de la "sequía extraordinaria", ya apuntado en las primeras páginas.

Por otra parte, en el acuerdo signado en la minuta 319 aparece el concepto de Agua Mexicana Creada Intencionalmente ([ICMA] Intentionally Created Mexican Allocation), que le permite tener una reserva en el lago Mead, para el caso de 
requerirla en años previos al 2018. En caso de no necesitarse, puede venderse en el mercado de transferencias de agua en Estados Unidos, sistema que opera en dicho país desde hace varias décadas. California, en lo específico es quizá la entidad que a partir de la década de 1970 ha generado una gran cantidad de acuerdos para realizar transferencias (Bauer, 2010).

\section{Prevención de futuro seco. Cambio conceptual de qué es variabilidad histórica}

Hemos planteado en este escrito que el tema del caudal variable fue sostenido desde principios del siglo xx. ¿Es la variabilidad de la corriente un efecto del cambio climático o es resultado de la evaporación en las presas, de las extracciones que eliminan las aguas de retorno? Como ya apuntamos, un autor como Parsons (1950) hace 68 años indicó que el río Colorado estaba sobre asignado. El discurso sobre el cambio climático ha permeado numerosas decisiones y ha generado una gran cantidad de propuestas y modificaciones en los escenarios a futuro.

En marzo de 2014, Sally Jewell, secretaria del Departamento del Interior de Estados Unidos y Juan José Guerra, en ese momento secretario del medio ambiente en nuestro país, realizaron declaraciones sobre las bondades de la minuta 319 , en las que se relacionan los temas de los cambios de asignación de agua a México, ya señalada, y los beneficios del documento en referencia al flujo que permitiría restaurar áreas del bajo delta del río Colorado (Jewell y Guerra, 2014). Dichas autoridades indicaron que “...los gobiernos de México y Estados Unidos hemos aprendido sobre los límites y la variabilidad del flujo del río Colorado, es por ello que estamos convencidos de que la mejor manera de enfrentar estos desafíos es la cooperación” (Jewell y Guerra, 2014, párr. 7).

Como se puede observar, en la nota parece que el tema de la variabilidad es algo que se ha aprendido más o menos recientemente, cuando en realidad —como es la tesis central de este artículo- tiene más de un siglo y ha sido punto central en diferentes momentos en que se han discutido las posibilidades de uso futuro de la corriente. Por otra parte, mientras algunas autoridades indicaron que sí se cambiaba el tratado de 1944, otros afirmaron en sentido contrario: no había modificación (Bono, 2012). En lo que a nosotros respecta, indicamos que queda ambiguo y que en la minuta se ratifica como referencia principal el tratado de 1944 y se señala expresamente que no es un precedente para futuros acuerdos. Dicho de otra manera, es el tratado internacional el que se va a cumplir, salvo la extensión de la minuta por así considerarlo conveniente para ambas partes. En ese sentido, en nuestra lectura, existe una posibilidad de mantener el acuerdo en relación con la altura de la presa Hoover hasta el año 2026.

\section{Para concluir}

Con lo anterior no pretendemos sumarnos a los argumentos que se realizan para negar el cambio climático (McCright y Dunlap, 2000). No es nuestra postura poner a tela de juicio el fenómeno global construido socialmente. Solo indicamos que esa variabilidad 
de río Colorado es un factor estudiado y reconocido desde las investigaciones realizadas a principios del siglo $\mathrm{xx}$.

Del recurso de dos ríos internacionales entre México y Estados Unidos —existen otros que aquí no tocamos- el Colorado y el Bravo, dependen alrededor de 60 millones de personas. Aquí solo tratamos el caso del Colorado que involucra a alrededor de 40 millones de habitantes. Si bien otros temas ocupan más espacio en la agenda bilateral, como la migración o el tráfico de drogas, el tema de los posibles conflictos por el agua se mantiene latente ante la interrogante ¿En qué momento el río podría ser modificado sustancialmente por el cambio climático? Por nuestra parte indicamos, la variabilidad ha sido muy amplia en determinados periodos, no solo como efecto del cambio climático.

La minuta 319 es una prevención en un marco donde las medidas ante el cambio climático es el discurso de mayor impacto. Por tanto, parece que los argumentos utilizados son una solución ante una nueva condición: el río Colorado es variable. Sin embargo, dicha condición es un tema de larga duración que aparece de manera reiterada.

\section{Referencias}

Adler, R. W. (2008). Revisiting the Colorado River Compact: Time for a Change? Journal of Natural Resources E Environmental Law, 28(1), 19-47.

Arellano, L. (13 de febrero de 2013). Combaten agricultores acta 319; se manifiestan en Mexicali [Mensaje en un blog]. Uniradio Informa. Recuperado de http:// www.uniradioinforma.com/noticias/bajacalifornia/174003/combaten-agricultores-acta-319-se-manifiestan-en-mexicali.html

Barnett, T., Malone, R., Peneell, W., Stammer, D., Stammer, B. y Washington, W. (2004). The Effects of Climate Change on Water Resources in the West: Introduction and Overview. Climatic Change, 62(1-3), 1-11. doi:10.1023/B:CLIM.0000013695.21726.b8

Bauer, C., J. (2010). El abanico de bancos de agua en las Américas. Estudios de Economía Aplicada, 28(2), 227-236.

Birdsong, B. C. (2011). Mapping the Human Right to Water on the Colorado River. Willamette Law Review, 48(117), 117-146.

Blanchard, R. (1924). Geographical Conditions of Water Power Development. The Geographical Review, XIV(1), 88-97.

Bono, R. M. (10 de diciembre de 2012). Minute 319: A Creative Approach to Modifying Mexico-U.S. Hydro-Relations Over the Colorado River [Mensaje de un blog]. Recuperado de http://www.internationalwaterlaw.org/blog/2012/12/10/minute-319

Bureau of Reclamation. (1946). The Colorado River. A natural menace becomes a national resource. A comprehensive report on the development of water resources of the Colorado river basin for irrigation, power production, and other beneficial uses in Arizona, California, Colorado, Nevada, New Mexico, Utah, and Wyoming. Estados Unidos de América: Department of Interior, the Bureau of Reclamation.

Christensen, N. y Lettenmaier, D. P. (2006). A Multimodel Ensemble Approach to Assessment of Climate Change Impacts on the Hydrology and Water Resources of the Colorado River Basin. Hydrology and Earth System Sciences, (11), 1417-1434. 
Colorado River Commission. (11 de noviembre de 1922). Minutes of the Eleventh Meeting. Santa Fe, Nuevo México, Estados Unidos de América.

Conway, C. J., Nadeau, C. P. y Piest, L. (2010). Fire Helps Restore Natural Disturbance Regime to Benefit Rare and Endangered Marsh Birds Endemic to the Colorado River. Ecological Applications, 20(7), 2024-2035.

Cook, B. I., Ault, T. R. y Smerdon, J. E. (2015). Unprecedented 21st Century Drought Risk in the American Southwest and Central Plains. Science Advances, 1(1), 1-7. doi: 10.1126 /sciadv.1400082

Cortez, A., Whiteford, S. y Chávez, M. (Coords.). (2005). Seguridad, agua y desarrollo. El futuro de la frontera México-Estados Unidos. Baja California, México: El Colegio de la Frontera Norte, Michigan State University.

Cruz-Castillo, M. (Abril de 2002). Catálogo de las fallas regionales activas en el norte de la Baja California, México. Geos, Unión Geofísica Mexicana, 37-42.

Enríquez, E. (1976). El tratado entre México y los Estados Unidos de América sobre ríos internacionales. Una lucha nacional de noventa años. México: Facultad de Ciencias Políticas y Sociales-Universidad Nacional Autónoma de México.

Fahlund, A., Choy, M. L. J. y Szeptycki, L. (2014). Water in the West. California Journal of Politics and Policy, doi:10.1515/cjpp-2013-0043, pp. 1-42

Farmer, J. (1996). Glen Canyon and the Persistence of Wilderness. The Western Historical Quarterly, 27(2), 210-222.

Forman frente común en defensa del agua del valle de Mexicali [Mensaje en un blog]. (16 de enero de 2013). Uniradio Informa. Recuperado de http://www.uniradioinforma.com/noticias/bajacalifornia/169229/forman-frente-comun-en-defensa-del-agua-del-valle-de-mexicali.html

Fox, J. (27 de abril de 2015). William Shatner's California Pipe Dream. Sending Washington's Water to California is DOA. Blomberg View. Recuperado de https:/ /www.bloomberg.com/view/articles/2015-04-27/william-shatner-s-california-pipe-dream

Glenn, E. P., Felger, R. S., Burquez, A. y Turner, D. S. (1992). Cienega de Santa Clara: Endangered Wetland in the Colorado River Delta, Sonora, Mexico. Natural Resources Journal, (32), 817-824.

Glenn, E. P., Garcia-Hernandez, J., Congdon, C., y Luecke, D. (1999). Status of Wetlands Supported by Agricultural Drainage Water in the Colorado River Delta, Mexico. Horticultural Science, 34(1), 16-21.

Glenn, E. P., Lee, C., Felger, R., y Zengel, S. (1996). Effects of Water Management on the Wetlands of the Colorado River Delta, Mexico. Conservation Biology, 10(4), 1175-1186.

Glenn, E., Tanner, R., Mendez, S., Kehret, T., Moore, D., Garcia, J., y Valdés, C. (1998). Growth Rates, Salt Tolerance and Water Use Characteristics of Native and Invasive Riparian Plants from the Delta of the Colorado River, Mexico. Journal of Arid Environments, 40(3), 281-294.

Gray, L. C. (1913). The Economic Possibilities of Conservation. The Quarterly Journal of Economics, 27(3), 497-518.

Haro, S. (1 de enero de 2017). Revive discusión por el agua. Semanario Zeta. Recuperado de http://zetatijuana.com/2017/01/01/revive-discusion-por-el-agua/

Harvey, M. W. T. (1991). Echo Park, Glen Canyon, and the Postwar Wilderness Movement. Pacific Historical Review, 60(1), 43-67. 
Hicks, W. (2007). Columbia River Diversion as a Public Revenue Source. (White paper). Recuperado de http://aquadoc.typepad.com/waterwired/files/Columbia_River_Revenue.pdf

Hundley, N. C. Jr. (1963). The Dividing Waters: Mexican-American Controversies Over the Waters of the Colorado River and The Rio Grande, 1880-1960. California, Estados Unidos de América: University of California.

Hundley, N. C. Jr. (1975). Water and the West. The Colorado River Compact and the Politics of Water in the American West. California, Estados Unidos de América: University of California Press.

Jewell, S. y Guerra, J. L. (31 de marzo de 2014). México y Estados Unidos, colaboración en el río Colorado. Milenio. Recuperado de http://www.milenio.com/firmas/ sally_jewell_y_juan_jose_guerra_abud_sally_jewell_y_juan_jose_guerra_abud/ Mexico-Unidos-colaboracion-rio-Colorado_18_272552781.html

Kelly, B. (3 de agosto de 2015). Drought relief, by rail. Railway Age. Recuperado de http://www.railwayage.com/index.php/freight/class-i/drought-relief-by-rail. html

La Rue, E. C. (1916). Colorado River and its Utilization (Water Supply Paper No. 395). Department of Interior, United States Geological Survey, Government Printing Office, Washington, Estados Unidos de América.

La Rue, E. C. (1925). Water, Power and Flood Control, of Colorado River Below Grenn River, Utah (Water Supply Paper No. 556). Department of Interior, United States Geological Survey Goverment Printing Office, Washington, Estados Unidos de América.

Luecke, D., Pitt, J., Congdon, C., Glenn, E., Valdés-Casillas, C. y Briggs, M. (1999). El delta una vez más: restaurando el hábitat ribereño y los humedales del delta del río Colorado. Washington, Distrito de Columbia, Estados Unidos de América: Enviromental Defense.

McCright, M. y Dunlap, R. (2000). Challenging Global Warming as a Social Problem: An Analysis of the Conservative Movement's Counter-Claims. Social Problems, 47(4), 499-522.

Muñoz, P. (18 de agosto de 2015). E. U. usufructúa agua del Valle de Mexicali: ccc. La jornada. Recuperado de http://www.jornada.unam.mx/2015/08/18/socie$\mathrm{dad} / 038 \mathrm{n} 2 \mathrm{soc}$

Nowak, K., Hoerling, M., Rajagopalan, B. y Zagona, E. (2012). Colorado River Basin Hydroclimatic Variability. Journal of Climate, 25(12), 4389-4403.

Olson, R. L. (1926). The Colorado River Compact (tesis de doctorado). Estados Unidos de América: Harvard University.

Overpeck, J., Garfin, G., Jardine, A., Busch, D. E., Cayan, D., Dettinger, M. ... y Udall, B. (2013). Summary for Decision Makers. En G. Garfin, A. Jardine, R. Merideth, M. Black, y S. LeRoy (Eds.), Assessment of Climate Change in the Southwest United States: A Report Prepared for the National Climate Assessment, (pp. 1-20). Washington, Distrito de Columbia, Estados Unidos de América: Island Press.

Parsons, M. B. (1950). Party and Pressure Politics in Arizona's Opposition to Colorado River Development. Pacific Historical Review, 19(1), 47-58.

Robison, J., Bratrschovsky, K., Latcham, J., Morris, E., Palmer, V. y Villanueva, A. (2014). Challenge and response in the Colorado River Basin. Water Policy, 16(s1), 12-57. 
Samaniego, M. A. (2006). Ríos internacionales entre México y Estados Unidos. Los tratados de 1906 y 1944. México: El Colegio de México, Universidad Autónoma de Baja California.

Samaniego, M. A. (2015). Usos sociales del agua y medio ambiente en la cuenca internacional del río Colorado, Región y Sociedad, (63), 61-96.

Sánchez, V. (2005). La demanda de agua en la región fronteriza México-Estados Unidos y los desafíos institucionales. En M. Chávez, S. Whiteford y A. A. Cortez (Coords.), Seguridad, agua y desarrollo. El futuro de la frontera México-Estados Unidos. México: El Colegio de la Frontera Norte.

Seavey, C. L. (1928). What the Boulder Dam Project Means to California and to the Nation. Annals of the American Academy of Political and Social Science, 135(1), 127-132.

Shor, G. G. Jr., Russell, W. R. y McGowan, D. D. (1976). Seismic Refractation Studies in the Southern California Borderland 1949-1974. Estados Unidos de América: University of California, San Diego Marine Physical Laboratory on the Scripps Institution of Oceanography.

Simon, S. (4 de mayo de 1990). Hahn Calls for an Aqueduct to the Northwest. Los Angeles Times. Recuperado de http://articles.latimes.com/1990-05-04/local/ me-181_1_columbia-river-water

Smith, T. G. (1995). John Kennedy, Stewart Udall, and New Frontier Conservation. Pacific Historical Review, 64(3), 329-362.

Squillace, M. (2012). Water Transfers for a Changing Climate (Working Paper Number 12-02). Estados Unidos de América: University of Colorado Law School.

Valdés-Casillas, C., Hinojosa-Huerta, O., Munoz-Viveros, M., Zamora-Arroyo, F., Carrillo-Guerrero, Y., Delgado-Garcia, S. ... y Luecke, D. (1998). Information Database and Local Outreach Program for the Restoration of the Hardy River Wetlands, Lower Colorado River Delta, Baja California and Sonora, Mexico. México: Instituto Tecnológico y de Estudios Superiores de Monterrey.

Work, H. (1925). [Hubert Work, Secretario del Interior a Frank B. Kellog, Secretario de Estado]. National Archives (Record group 115, entry 7, caja 36 032.5), Denver, Colorado, Estados Unidos de América.

\section{Material legislativo}

Arizona vs California, 207 U.S. 564 (1963).

Arizona vs California, 373 U.S. 546 (1963).

Boletín 1398. Pide senado proveer de suficiente agua de riego a campesinos del valle de Mexicali, 18 de abril de 2013. Recuperado de http://comunicacion.senado. gob.mx/index.php/informacion/boletines/6554-boletin-1398-pide-senadoproveer-de-suficiente-agua-de-riego-a-campesinos-del-valle-de-mexicali.html

Boulder Canyon Reclamation Project. 1926. Committee on irrigation and reclamation, report 3331, rep. 654, part 1 [Senate 66 congress].

Colorado River Development. Colorado River investigations water storage and power development Grand Canyon to the Imperial Valley 1928, 70 congress session, documento 180, [Senado de los Estados Unidos, Goverment Printing Office]. 
Dictamen de acuerdo del congreso del estado de Baja California por el que se exhorta a la celebración de una mesa de trabajo en relación con la firma del acta 319 entre México y Estados Unidos. 9 de abril de 2013. Recuperado de http://sil. gobernacion.gob.mx/Archivos/Documentos/2013/04/asun_2967003_201304 16_1366122205.pdf

Hearings before the subcomitte on irrigation and reclamation, H.R. 4671 and similar bills to authorize the construction, operation, and maintenance of the lower Colorado River basin Project [89 congress, Senado de los Estados Unidos]. Agosto-septiembre de 1965 .

Hearings before the subcomitte on irrigation and reclamation, H.R. 3300 and similar bills to authorize the construction, operation, and maintenance of the lower Colorado River basin Project. [90 congress, Senado de los Estados Unidos]. Marzo de 1967.

Minute No. 317 Conceptual Framework for U.S.-Mexico Discussions on Colorado River Cooperate Actions, International Boundary and Water Comission. United States and México. 17 de junio de 2010. Recuperado de https:/ /www.ibwc.gov/ Files/Minutes/Minute_317.pdf

Minute No. 318 Adjustment of Delivery Schedules for Water Alloted to Mexico for the Years 2010 Through 2013 as a Result of Infraestructure Damage in Irrigation District 014, Rio Colorado, Caused by the April 2010 Earthquake in the Mexicali Valley, Baja California. International Boundary and Water Comission. United States and México. 17 de diciembre de 2010. Recuperado de https:/ /www.ibwc. gov/Files/Minutes/Min_318.pdf

Minute No. 319 Interim International Cooperative Measures in the Colorado River Basin Through 2017 and Extension of Minute 318 Cooperative Measures to Adress the Continued Efects of the April 2010 Earthquake in the Mexicali Valley, Baja California, International Boundary and Water Comission. United States and México. 20 de noviembre de 2012. Recuperado de https:/ / www.ibwc.gov/Files / Minutes/Minute_319.pdf

Minute No. 319 of the International Boundary and Water Comission, United States of America and Mexico. Boundary Waters. Threaties and Other International Acts Series 12-1127. [Department of State]. 20 de noviembre de 2012. Recuperado de https://www.state.gov/documents/organization/205768.pdf

National Archives Denver, Record Group 115, caja 2764 B. Denver, Colorado, Estados Unidos de América.

Water Treaty with Mexico 1945 [Comité de Relaciones Exteriores del Senado de los Estados Unidos, Government Printing Office].

Marco Antonio Samaniego López

Mexicano. Doctor en historia por El Colegio de México. Investigador del Instituto de Investigaciones Históricas de la Universidad Autónoma de Baja California (UABC). Licenciado en Pedagogía y licenciado en Historia (UABC). Líneas de investigación: la frontera México-Estados Unidos como construcción social, historia y memoria colectiva, historia de Baja California y sus relaciones con los espacios fronterizos. Publicaciones recientes: El Bureau of Reclamation: los cambios de sus políticas hidráulicas y ambientales y su relación con la Comisión Internacional de Límites y Aguas, en Cuencas Transfronterizas (2017), y El poblado fronterizo de Tijuana, Emiliano Zapata y la revoluzione da tavolino, en Historia Mexicana (2017). 